\title{
Intranasal Insulin Reduces White Matter Hyperintensity Progression in Association with Improvements in Cognition and CSF Biomarker Profiles in Mild Cognitive Impairment and Alzheimer's Disease
}

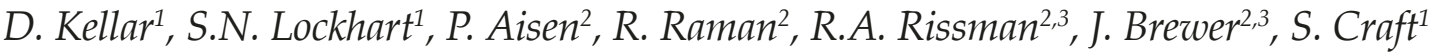

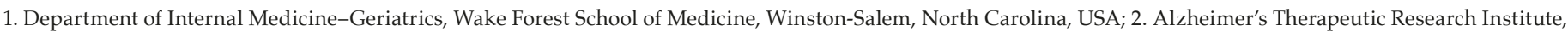 \\ University of Southern California, San Diego, USA; 3. Department of Neurosciences, University of California, San Diego, La Jolla, USA
}

Corresponding Author: Suzanne Craft, PhD, Department of Internal Medicine-Geriatrics, Wake Forest School of Medicine, One Medical Center Boulevard, WinstonSalem, NC 27157, suzcraft@wakehealth.edu

\begin{abstract}
BACKGROUND: Intranasally administered insulin has shown promise in both rodent and human studies in Alzheimer's disease; however, both effects and mechanisms require elucidation.

OBJECTIVE: We assessed the effects of intranasally administered insulin on white matter health and its association with cognition and cerebral spinal fluid biomarker profiles in adults with mild cognitive impairment or Alzheimer's disease in secondary analyses from a prior phase 2 clinical trial (NCT01767909).

DESIGN: A randomized (1:1) double-blind clinical trial.

SETTING: Twelve sites across the United States.

PARTICIPANTS: Adults with mild cognitive impairment or Alzheimer's disease.

INTERVENTION: Participants received either twice daily placebo or insulin (20 IU Humulin R U-100 b.i.d.) intranasally for 12 months. Seventy-eight participants were screened, of whom 49 (32 men) were enrolled.

MEASUREMENTS: Changes from baseline in global and regional white matter hyperintensity volume and gray matter volume were analyzed and related to changes in cerebral spinal fluid biomarkers, Alzheimer's Disease Assessment ScaleCognition, Clinical Disease Rating-Sum of Boxes, Alzheimer's Disease Cooperative Study-Activities of Daily Living Scale, and a memory composite.

RESULTS: The insulin-treated group demonstrated significantly reduced changes in white matter hyperintensity volume in deep and frontal regions after 12 months, with a similar trend for global volume. White matter hyperintensity volume progression correlated with worsened Alzheimer's disease cerebral spinal fluid biomarker profile and cognitive function; however, patterns of correlations differed by treatment group. Conclusion: Intranasal insulin treatment for 12 months reduced white matter hyperintensity volume progression and supports insulin's potential as a therapeutic option for Alzheimer's disease.
\end{abstract}

Key words: Alzheimer's disease, clinical trial, intranasal insulin, white matter, CSF.

Received February 15, 2021

Accepted for publication March 9, 2021

\section{Introduction}

lzheimer's disease (AD) is the leading cause $\triangle$ of dementia and, as there are currently no 1 disease modifying treatments, its prevalence is expected to increase in response to an aging population (1). AD is characterized by aggregation of amyloid beta (A $\beta$ ) plaques and tau neurofibrillary tangles (NFT). Clinical trials have attempted to reduce accumulation of these proteins in the brain and prevent further cognitive decline; however, although some amyloid antibody trials have successfully reduced plaque load, none have been successful to date in halting the progression of $\mathrm{AD}$ symptoms $(2,3)$. Positron Emission Tomography (PET) measures $A D$ pathology by quantifying the load of $A \beta$ and NFT in the brain, and a number of studies have demonstrated relationships between PET measures and concentrations of these proteins in cerebral spinal fluid (CSF) $(4,5)$. CSF A 342 decreases as amyloid accumulation in brain increases, suggesting it is being sequestered in the brain parenchyma, while hyperphosphorylated tau (p-tau) in CSF increases with increased propagation of NFT (6). Utilizing ratios of $A \beta 42$ to $A \beta 40$, p-tau, and total tau (t-tau) has been found to further improve specificity and sensitivity to identify $\operatorname{AD}$ dementia $(7,8)$. There remains a clear need for a pharmacological intervention to prevent or slow $\mathrm{AD}$ progression.

As AD progresses, gray matter volume is reduced. While this is also true of non-pathological aging, adults with AD exhibit a far greater reduction in overall gray matter associated with a predictable pattern (9). The hippocampus and entorhinal cortex are perhaps the most easily detected regions affected by AD (10); however, an $\mathrm{AD}$-defined meta region including the hippocampus and other regions such as entorhinal, inferior temporal, middle temporal, inferior parietal, fusiform, and precuneus has recently been utilized in order to increase sensitivity (11). Cortical thickness has also been postulated to be a more reliable marker of $\mathrm{AD}$ progression as it is less affected by total intracranial volume which can vary greatly between patients (11). While progression of the disease and corresponding 
gray matter reduction have been well established, its utility as a clinical trial endpoint needs to be validated. Interventions that have successfully removed amyloid or even allayed cognitive decline have also been associated with reduced gray matter volume $(12,13)$. These findings illustrate a complex relationship between brain health and gray matter volumetrics which is not fully understood.

White matter integrity can be reflected by the presence of white matter hyperintensities (WMH) detected with magnetic resonance imaging (MRI); however, the relationship of WMHs and AD progression has not been characterized in detail. WMHs are detected using fluid-attenuated inversion recovery (FLAIR) MRI, are presumed to indicate cerebrovascular pathology (14), and are associated with gliosis, demyelination, axonal loss, and arteriosclerosis (15). It is postulated that WMHs reflect a number of factors including hypoxia, amyloid angiopathy, blood brain barrier damage, degeneration, hypoperfusion, and inflammation (15). WMH volume (WMHV) increases with age and some studies have found WMHV to be independent of $A \beta$ burden, leading to the proposal that WMHs should be considered a co-pathology that do not directly contribute to $\mathrm{AD}$ $(16,17)$. Other studies have found correlations between WMHs and cortical tau load in AD (18). It has been proposed that cerebrovascular pathology represented by $\mathrm{WMHs}$ precedes and therefore could initiate $\mathrm{A} \beta$ aggregation (19-21). Conversely, other investigators claim that $A \beta$ induces vascular damage through neuroinflammation, formation of reactive oxygen species, and oxidative stress $(22,23)$. It is possible that vascular and $\mathrm{AD}$-specific pathology form a vicious cycle giving rise to these differing viewpoints, or that the precise time course of the association differs for subgroups of patients with AD.

Identification of the nature of the association of WMHs and AD is hindered by lack of a commonly-accepted standardized approach to measurement of WMHs (24). Methods evaluating WMHs range from semi-quantitative visual reads using one of 3 established rating scales (Manolio, Fazekas and Schmidt, Scheltens) to fully automated lesion segmentation $(25,26)$. Representation of the data is also not consistent, with some studies reporting global ratings or volumes and others focusing on spatial patterns $(27,28)$. There are also many ways to spatially segment WMHs such as periventricular versus deep (29); however, deep can also be further split in to juxtacortical and non-juxtacortical[30]. Studies in which WMHs are segmented in classical lobular fashion have reported that $\mathrm{AD}$ is associated with temporal WMHs (31), or with global and parietal/occipital volumes (32). Volumes can also be displayed as raw volumes (33), log transformed values (33), or percent change ratios $(34,35)$. As the field progresses automated techniques generating quantitative spatially accurate information may prove the best way to track WMH progression in $\mathrm{AD}$. It is clear that WMHs are associated with poor cognitive outcomes and preventing progression is a clinically relevant marker.
A promising area of research in the treatment and prevention of $\mathrm{AD}$ focuses on metabolism, inflammation and, in particular, the role of insulin in the central nervous system. With respect to metabolism, although insulin does not appear to impact global transport of glucose into the brain, it has been shown to increase glucose uptake via the glucose transporter GLUT4 in selected regions such as the hippocampus (36). Further, insulin increases glycogen storage in astrocytes, thereby providing an alternate energy source during glucose deprivation or intense neuronal activity (37). Insulin has long been implicated in $\mathrm{AD}$ and several reviews have highlighted both its importance and therapeutic potential (38-40). In short, insulin has been demonstrated to modulate both $\mathrm{A} \beta$ and pathological tau formation, and improves neuronal health, dendritic spine proliferation, and white matter integrity. Insulin can be administered intranasally where it is detectible in perivascular spaces with PET imaging (41) and in the CSF in less than 30 minutes (42). A promising pilot trial documented improvements in delayed memory recall, preserved Alzheimer's Disease Assessment Scale (ADAS-Cog) scores, and functional abilities assessed by the Alzheimer's Disease Cooperative Study-Activities of Daily Living Scale (ADCS-ADL) after 4 months of treatment with intranasal insulin compared to placebo (43). A recent large 18-month phase II clinical trial of INI treatment in AD and MCI patients found differing patterns of results depending on the device used to administer the insulin (13). For the device used by the primary intent-to-treat cohort, no significant differences in rates of decline measured by the ADAS-Cog13, Clinical Disease Rating-Sum of Boxes (CDR-SOB), ADCS-ADL, or CSF $A \beta$ and tau were observed between placebo and insulin groups. In a secondary cohort, a different device showed better performance on ADAS-Cog13 in the insulin-treated group compared to placebo at 6 months with a similar trend at 12 months. In open-label analyses, the early-start secondary device cohort treated with insulin performed better on the ADAS-Cog13 and ADL-MCI at 18 months than the delayed start secondary group. The insulin-treated group using this device also demonstrated an improvement in CSF A $\beta 42 / A \beta 40$ and $\mathrm{A} \beta 42 / \mathrm{t}$-tau ratios at 12 months. This study highlights the need for additional investigation to definitively determine the potential for intranasal insulin as a therapeutic for $\mathrm{AD}$.

In the present study, we assessed the effects of INI on white matter health in the secondary cohort of participants using the device associated with improved cognition and AD biomarker profiles. There are several mechanisms through which insulin could act directly to improve white matter health and prevent $\mathrm{WMH}$ progression (44). Reduced insulin levels or activity impair oligodendrocyte myelin survival and maintenance, and increase ceramides and decreases sulfatides, leading to oxidative stress, inflammation, and lipid peroxidation. These factors all contribute to myelin damage and subsequent WMHs. Insulin resistance impairs vascular 
responsiveness, causing luminal narrowing and fibrosis, which cause decreased blood flow and blood brain barrier damage. These effects lead to ischemia and inflammation and promote the formation of WMHs. As mentioned previously, insulin reduces $A \beta$ and p-tau levels in the brain, both of which can cause inflammation, neuronal and glial damage, and vascular impairment (45). These distinct pathways could all culminate in the formation and progression of WMHs, thus poising insulin at a convergence point in several potential cascades, and raising the possibility that providing insulin to the brain to overcome deficient insulin availability or activity may have therapeutic benefit in AD.

Based on this evidence, we examined the effect of 12 months of INI treatment vs. placebo on change in WMHs. We also examined the relationships among changes in WMHs, cognition, and AD CSF biomarkers.

\section{Methods}

The study was overseen by the Alzheimer's Therapeutic Research Institute (P. Aisen, Director) together with the Principal Investigator (S. Craft). Eligibility and recruitment for this study have been described previously (13). Two devices were used in the parent study; however, only one device demonstrated cognitive benefits or changes in AD CSF biomarkers across the 18-month long study. For this reason, we evaluated only the group using the device that showed potential beneficial effects. Briefly, participants with AD $(n=31)$ or amnestic MCI $(n=18)$ were recruited from 12 sites. Participants received baseline testing including CDR, MMSE, ADAS-Cog13, a lumbar puncture, and an MRI, then were randomized on a 1:1 basis to receive either 20 IU intranasal insulin $(n=24)$ or placebo $(n=25)$ twice daily for 12 months. After 12 months the cognitive battery was readministered, a lumbar puncture was performed, and another MRI was obtained. There were a total of 40 participants (insulin $n=20$; placebo $n=20$ ) with MRI data that passed quality control measures at baseline and month 12 .

T1 and Fluid Attenuated Inversion Recovery images were collected with 1.5 or 3T MRI. T1 weighed images were processed using FreeSurfer 6.0.0 to produce participant specific gray matter volume, thickness, and area. FLAIR images were segmented by the lesion growth algorithm[46] as implemented in the LST toolbox version 3.0.0 (www.statisticalmodelling.de/lst.html) for SPM. The algorithm first segments the T1 images into the three main tissue classes (CSF, GM and WM). This information is then combined with the coregistered FLAIR intensities in order to calculate lesion belief maps. By thresholding these maps with a pre-chosen initial threshold $(\kappa=0.3)$ an initial binary lesion map is obtained which is subsequently grown along voxels that appear hyperintense in the FLAIR image. The result is a lesion probability map. The lesion probability maps were then warped to MNI space and lobular volume was extracted using Mayo Clinic Adult Lifespan Template (47). A temporal-parietal volume meta-ROI was created to examine volume and was defined as bilateral entorhinal, inferior temporal, middle temporal, inferior parietal, fusiform, and precuneus (11). Cortical thickness was similarly defined by Jack (48) as the surface area weighted thickness of the entorhinal, inferior temporal, middle temporal, and fusiform.

CSF was collected in the morning after an overnight fast and was immediately placed on dry ice and was shipped overnight to the central biomarker laboratory. $\mathrm{AD}$ biomarkers $\mathrm{A} \beta 42, \mathrm{~A} \beta 40$, total tau, and tau phosphorylated at threonine 181 were quantified with the Meso Scale Discovery platform (Meso Scale Diagnostics). Blood was collected for APOE genotyping using established protocols.

Cross sectional analysis was performed to assess group differences at baseline in age, cognitive status, sex, baseline surface weighted cortical thickness, AD signature region volume, total $\mathrm{WMHV}$, and regional WMHV at baseline using general linear models or chi squared tests when appropriate. Change variables for gray matter and WMHV were defined as percentage change from baseline as previously described (35). General linear modeling was performed in SAS v 9.4 with covariates age, ApoE4 status, study site, and sex included in all initial models. Baseline volumes were also included for GMV and WMHV models, and total intracranial volume was included in models analyzing data in native space. Non-contributing covariates $(p>0.15)$ were dropped from the model. No adjustments were made for multiple comparisons; rather, results are reported as mean estimates and corresponding 95\% confidence intervals. In exploratory analyses, we examined whether individual treatment groups showed reliable change in WMHV over time with within-group LSMEANS t-tests. Change variables for WMHV, cognitive scores, and CSF values were subjected to Pearson's r correlations to determine inter-relationships.

\section{Results}

\section{Participants}

For the parent study secondary cohort that utilized the device associated with cognitive benefit, 78 participants were screened, of whom 49 (32 men [65.3\%]) were enrolled. Twenty-four were randomized to the insulin arm and 25 were randomized to the placebo arm (Figure 1 ). Of those 49,40 participants (insulin $n=20$, placebo $\mathrm{n}=20$ ) had usable MRI data at both time points and were analyzed for this study. There were no demographic or other notable clinical differences between participants with usable and unusable data. There were also no differences in demographic characteristics between arms at baseline (Table 1 ). 


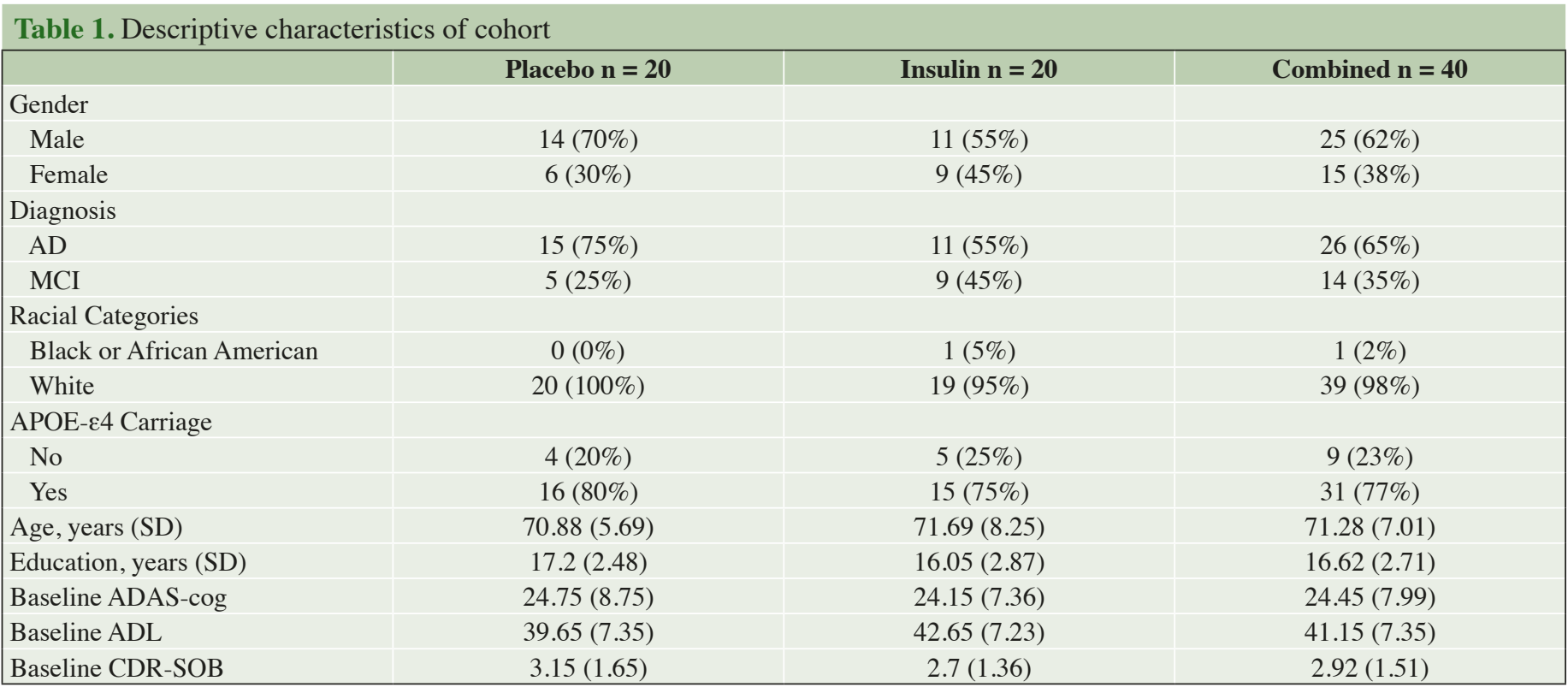

\section{Figure 1. CONSORT diagram}

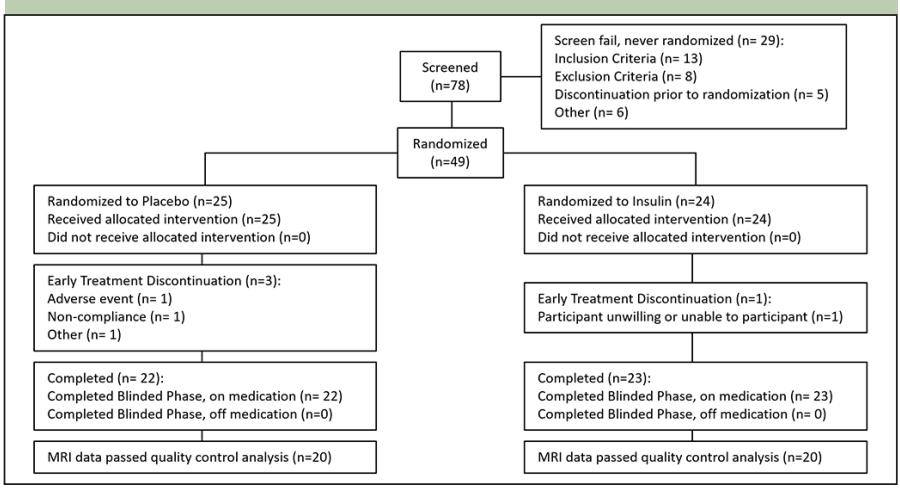

\section{MRI Results}

The temporal-parietal meta-ROI decreased in volume over time as did the surface weighted cortical thickness (both ps<.001, Figure 2). There was no interaction between treatment arm and rate of decline of gray matter or cortical thickness (Supplementary Table 1).

An interaction between treatment arm and global WMHV was observed such that the insulin-treated arm tended to have less global WMH volume increase over the 12 month intervention compared to the placebo group (insulin lsmeans [95\% CI] $=18.98[-1.38,39.33]$ and placebo 42.21 [21.70,62.72], p=0.064, Figure 3). Given this trend, exploratory analyses were conducted for comparisons of individual ROIs between treatment arms. Insulin significantly reduced WMHV change over the 12 month intervention in both the frontal lobe and deep white matter compared to placebo (frontal insulin lsmeans $[95 \% \mathrm{CI}]=15.14[-3.84,34.12]$ and placebo $=39.18$ $[20.05,58.30], \mathrm{p}=0.042$; deep WM insulin lsmeans [95\% $\mathrm{CI}]=56.94[-20.20,134.08]$ and placebo $=161.37[81.68$, 241.05], $p=0.042$, Figure 3). Change in WMHV was less in the insulin arm than the placebo arm for all other regions, although these comparisons did not reach statistical significance.

When we examined whether individual treatment groups showed reliable change in WMHV over time with within-group LSMEANS t-tests, the placebo group showed significantly increased WMHVs across all regions (all ps $<0.05$, Figure 3; raw means for baseline and month 12 for all regions are presented in Supplementary Table 2), whereas WMHV was unchanged following insulin treatment in the deep white matter, corpus callosum, occipital, parietal, and frontal regions (all $p>0.1$, Figure 3 ). Temporal WMHV increased slightly over the 12 month intervention with insulin treatment with a similar trend in global WMHV (temporal $\mathrm{p}=0.033$; global $\mathrm{p}=0.066$ ), although to a lesser degree than with placebo.

\section{Correlation between MRI and Cognitive Outcomes}

For the combined cohort including both insulin and placebo groups, increased global WMHV correlated with lowered memory composite scores $(\mathrm{r}=-0.38, \mathrm{p}=0.024$, Figure $4 \mathrm{~A}$ ) and similar trends were observed for the ADAS-Cog13 and CDR-SOB ( $r=0.297, p=0.062 ; r=0.278$, $\mathrm{p}=0.081$, figure 4A). Regional analysis revealed a significant correlation between the memory composite score and parietal and occipital WMHVs and a trend correlation for the corpus callosum $(\mathrm{r}=-0.536, \mathrm{p}=0.001$; $r=-0.405, p=0.015 ; r=0.31, p=0.069$, Figure 4A). Increased temporal WMHV was associated with worsened (higher) scores for the ADAS-Cog13 $(r=0.313, p=0.049$, Figure 4A). A similar trend correlation was observed between frontal WMHV and ADCS-ADL scores $(r=-0.267, p=0.095$, Figure $4 \mathrm{~A})$.

When analyzed by treatment group, the insulin group showed a significant correlation between change in ADAS-Cog13 scores and WMHVs change in the corpus callosum, and trending correlations for the deep white matter and temporal regions $(\mathrm{r}=0.459, \mathrm{p}=0.041$; 
$\mathrm{r}=0.409, \mathrm{p}=0.073 ; \mathrm{r}=0.368, \mathrm{p}=0.092$, Figure 4B). Increased (worsened) CDR-SOB scores correlated significantly with increased frontal and global WMHV with trends noted for deep white matter, temporal, and the corpus callosum regions $(\mathrm{r}=0.535, \mathrm{p}=0.014 ; \mathrm{r}=0.537, \mathrm{p}=0.014 ; \mathrm{r}=0.439$, $\mathrm{p}=0.052 ; \mathrm{r}=0.426, \mathrm{p}=0.059 ; \mathrm{r}=0.409, \mathrm{p}=0.061$, Figure $4 \mathrm{~B}$ ). Smaller increases in frontal WMHV also correlated with smaller increases in ADCS-ADL scores ( $\mathrm{r}=-0.475, \mathrm{p}=0.034)$. In the placebo group, declines in memory composite scores only correlated with increased WMHV in global, parietal, and occipital regions with trends for frontal, corpus callosum, and temporal regions $(\mathrm{r}=-0.639, \mathrm{p}=0.005$; $\mathrm{r}=-0.773, \mathrm{p}=0.001 ; \mathrm{r}=-0.585, \mathrm{p}=0.013 ; \mathrm{r}=-0.48, \mathrm{p}=0.05$; $\mathrm{r}=0.435, \mathrm{p}=0.08 ; \mathrm{r}=-0.425, \mathrm{p}=0.088$, Figure $4 \mathrm{C}$ ).

Figure 2. Changes in gray matter A) volume and B) surface weighted thickness in the temporal-parietal meta-ROI

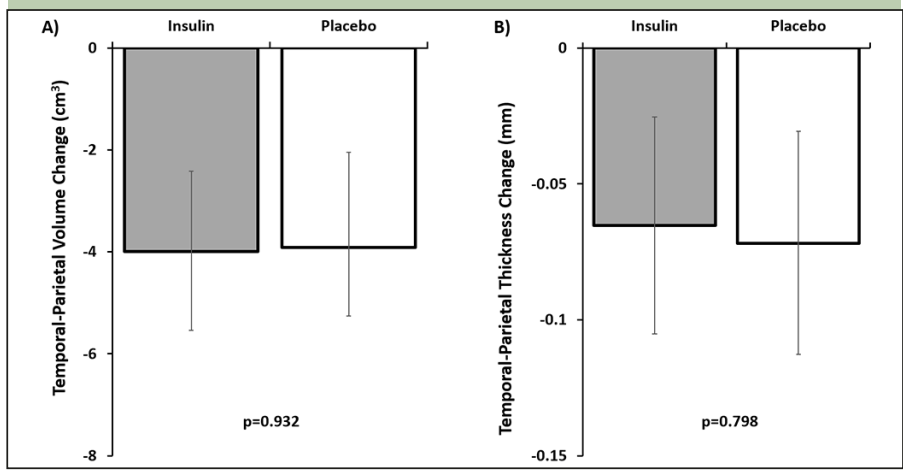

There were no significant differences between treatment group and placebo. Error bars represent $95 \%$ confidence intervals.

\section{Correlation between MRI and CSF outcomes}

For the combined cohort including both insulin and placebo groups, global WMHV increase correlated with a decrease in CSF A $\beta 42$ and with a similar trend for the
$\mathrm{A} \beta 42$ / $\mathrm{A} \beta 40$ ratio $(\mathrm{r}=-0.375, \mathrm{p}=0.028 ; \mathrm{r}=-0.328, \mathrm{p}=0.058$, Figure 5A). Frontal WMHV increases also correlated with decreases in both $A \beta 42$ and $A \beta 42 / A \beta 40$ ratio ( $r=-$ $0.355, \mathrm{p}=0.039 ; \mathrm{r}=-0.44, \mathrm{p}=0.009$, Figure $5 \mathrm{~A})$. Increases in both corpus callosum and deep white matter WMHV correlated with decreases in $A \beta 42 /$ tau ratio ( $r=-0.397$, $\mathrm{p}=0.001 ; \mathrm{r}=-0.734, \mathrm{p}=0.001$, figure $5 \mathrm{~A}$ ).

When analyzed by treatment group, there was a significant correlation between increased parietal WMHV and decreased $A \beta 40$ in the insulin group $(r=-0.525$, $p=0.036$, figure $5 B$ ). There were trending relationships between increased global WMHV and decreased A $\beta 42$ / $\mathrm{A} \beta 40$ ratio, increased frontal WMHV and decreased $\mathrm{A} \beta 42 / \mathrm{A} \beta 40$ ratio and $A \beta 42 / \mathrm{t}$-tau ratio changes, and increased corpus callosum and decreased $A \beta 42$ levels $(\mathrm{r}=-0.443, \mathrm{p}=0.085 ; \mathrm{r}=-0.467, \mathrm{p}=0.067 ; \mathrm{r}=-0.477, \mathrm{p}=0.061$; $\mathrm{r}=-0.446, \mathrm{p}=0.083$, figure $5 \mathrm{~B})$. In the placebo group, increases in both corpus callosum and deep white matter WMHV correlated with decreases in $\mathrm{A} \beta 42 / \mathrm{t}$-tau ratio ( $\mathrm{r}=-$ $0.733, \mathrm{p}=0.001 ; \mathrm{r}=-0.724, \mathrm{p}=0.001$, figure $5 \mathrm{C}$ ). Temporal WHV change positively correlated with ptau-181/t-tau ratio $(\mathrm{r}=0.481, \mathrm{p}=0.043$, figure $5 \mathrm{C})$. There was a trend for both global and frontal WMHV change to negatively correlate with $\mathrm{A} \beta 42$ change $(\mathrm{r}=-0.431, \mathrm{p}=0.073 ; \mathrm{r}=-0.408$, $\mathrm{p}=0.092$, figure $5 \mathrm{C}$ ).

\section{Discussion}

The present study found that increased WMHV correlated with greater declines in cognition and worsening of CSF AD biomarker profiles, and that INI treatment for 12 months reduced WMHV progression in key brain regions. White matter hyperintensities represent cerebral small vessel disease and white matter damage resulting from degraded myelin, and have been shown to increase with aging. Some studies have suggested that regional, and not global WMHV may best

Figure 3. White Matter Hyperintensity Volume (WMHV) as percent change from baseline both globally and regionally split by MCALT (excluding cerebellum and midbrain regions)

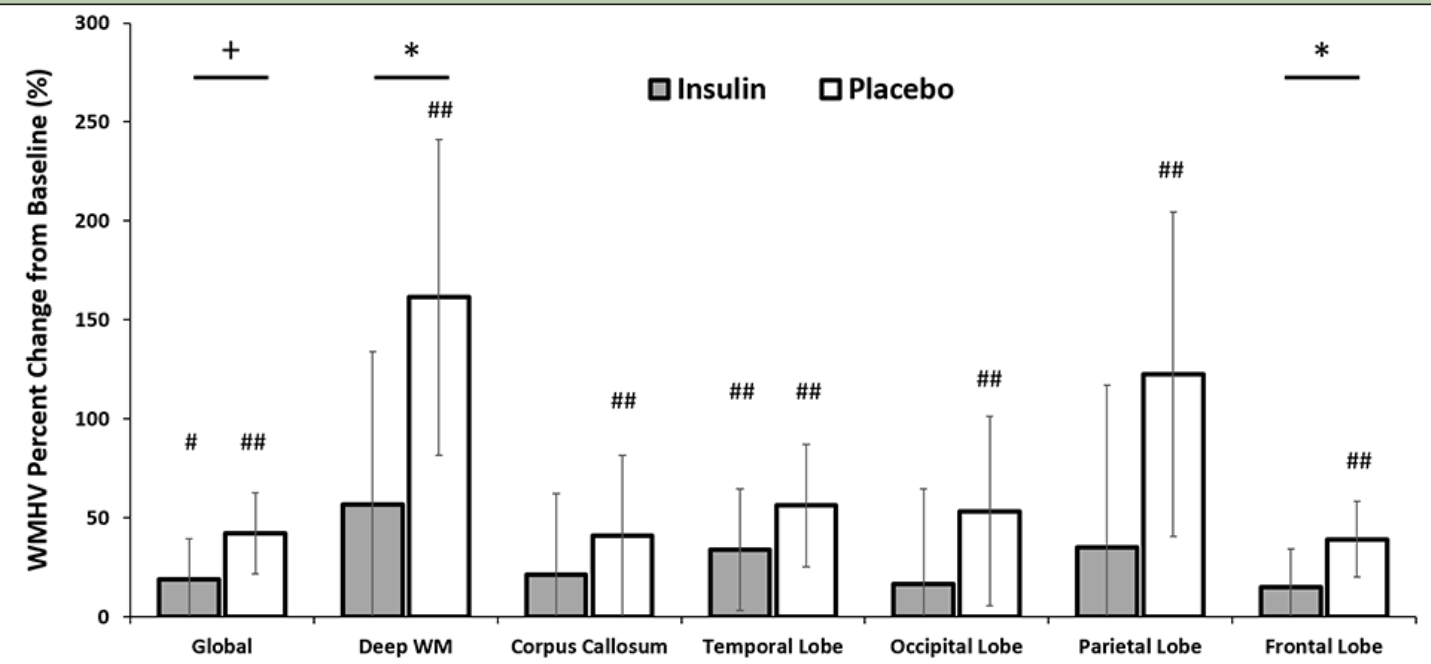

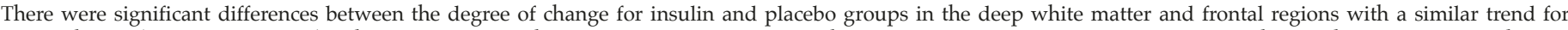

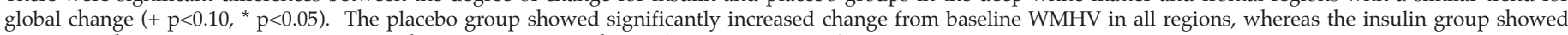
significant change only in temporal lobe with a trend for global change $(\#<0.10$, \#\# $\mathrm{p}<0.05)$. Error bars represent $95 \%$ confidence intervals. 
Figure 4. Changes in global and regional White Matter Hyperintensity Volume (WMHV) correlate with changes in ADAS-cog, CDR-SB, ADCS-ADL, and a memory composite

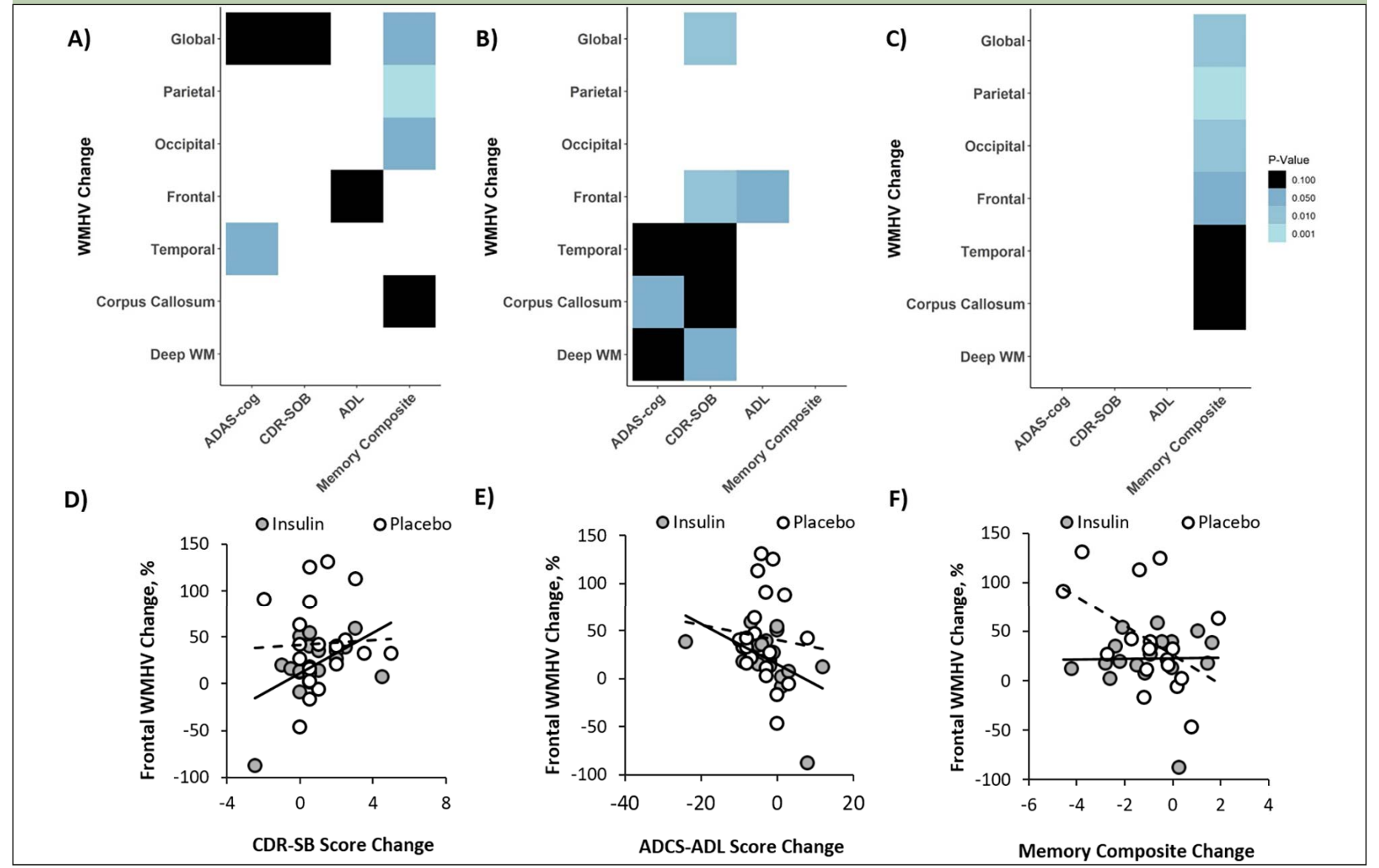

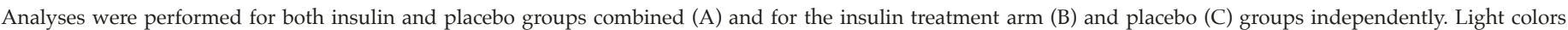

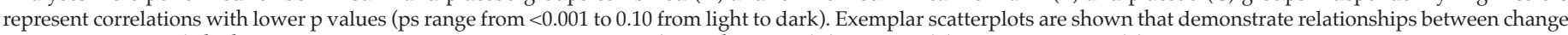
in frontal WMHVs (which differed between insulin and placebo groups) and change in (D) CDR-SB, (E) ADCS-ADL, and (F) memory composite scores.

predict, or correlate, with AD progression (49). While there are numerous ways to segregate WMHs the most promising research has suggested a division between frontal and posterior regions in differentiating normal versus pathological aging, with lobular divisions further increasing the regional specificity $(32,50)$. We observed that INI treatment for 12 months slowed the progression of WMHs globally and in deep WM and frontal regions compared to placebo assignment. This finding supports previous studies linking frontal WMHs and pathological aging and suggests that INI may reduce AD-related WMH progression in these key areas. Exploratory analysis also demonstrated that for all other regions, with the exception of the temporal lobe, progression of WMHs were stabilized following insulin treatment. These findings may be considered clinically significant as growing research has shown that WMHVs correlate with numerous pathological outcomes such as ependymal loss, cerebral ischemia, and demyelination (51). Insulin could be acting through a number of pathways to preserve white matter health (44). Insulin resistance impairs oligodendrocyte myelin maintenance and survival, while also leading to reduced vascular smooth muscle responsiveness. Increasing insulin availability could ameliorate these deficiencies thereby reducing $\mathrm{WMH}$ progression.

Previous research has shown that higher baseline WMHV predicts worse cognitive outcomes measured by the ADAS-Cog (52) and CDR (53). We demonstrated in the combined cohort that over 12 months, longitudinal increases in WMHV correlate with decreases in cognition measured by the ADAS-Cog, CDR-SOB, ADCS-ADL, and a memory composite. We also found that correlations differed depending on treatment group. The placebo group showed correlations only between WMHV and memory composite scores, while the insulin treated group showed associations between WMHs and both the ADASCog13 and CDR-SOB, as well as the ADCS-ADL. Of note, in the parent study, insulin treatment was shown to benefit performance on the ADAS-Cog13, CDR, and ADCS-ADL for the secondary cohort (13). The present findings raise the possibility that insulin's effects on these measures are mediated in part by factors associated with WMHV. These findings suggest that preventing or even delaying progression of white matter damage could prevent global cognitive and functional decline.

Increased CSF A $\beta 42$ levels and decreased hyperphosphorylated tau concentrations have been 
Figure 5. Changes in global and regional White Matter Hyperintensity Volume (WMHV) correlate with changes in CSF AD biomarkers

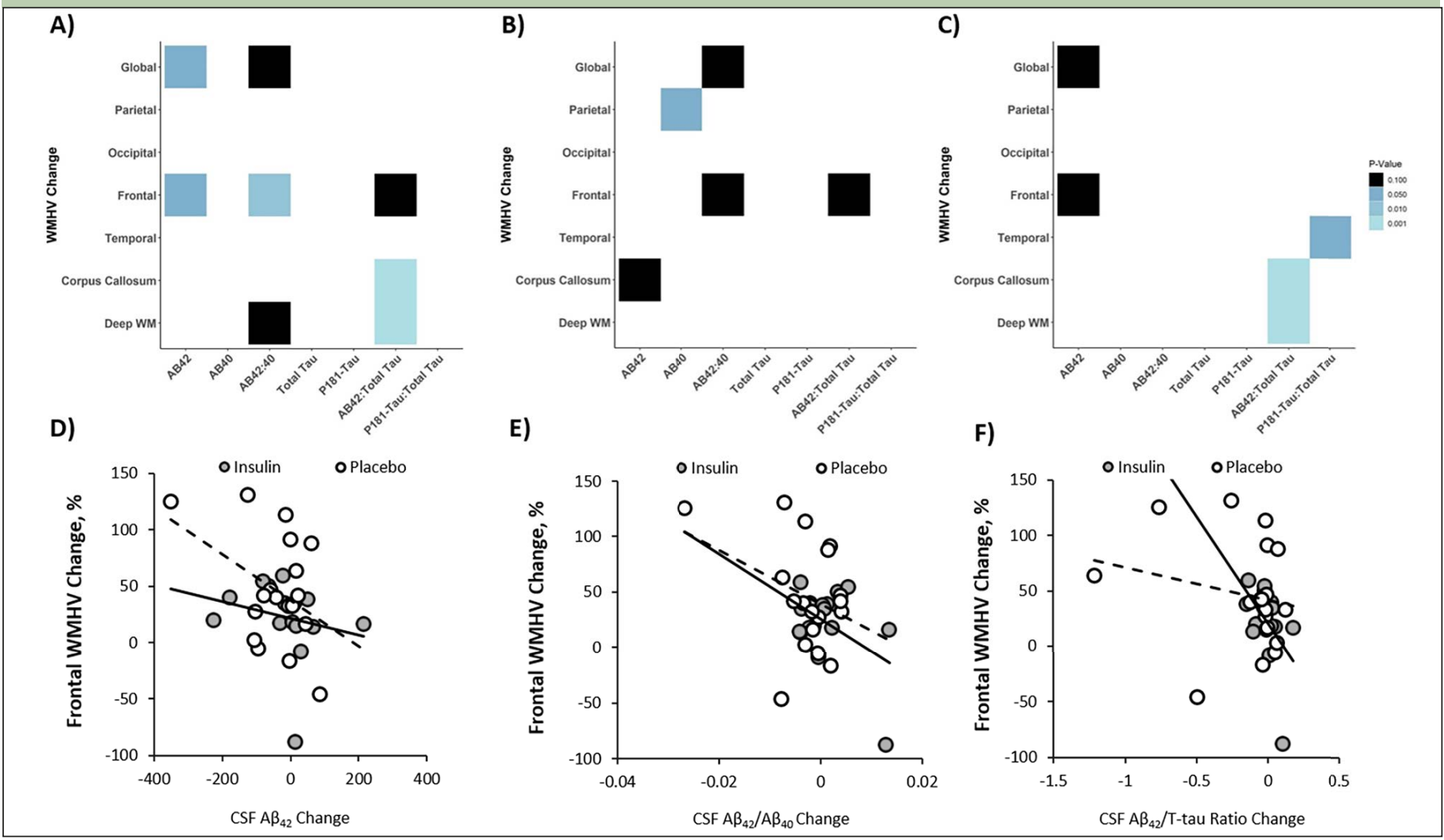

Analyses were performed for both insulin and placebo groups combined (A) and for the insulin treatment arm (B) and placebo (C) groups independently. Light colors represent correlations with lower $\mathrm{p}$ values ( $\mathrm{ps}$ range from $<0.001$ to 0.10 from light to dark). Exemplar scatterplots are shown that demonstrate relationships between change in frontal WMHVs (which differed between insulin and placebo groups) and change in CSF (D) A $\beta 42$, (E) A $\beta 42 / A \beta 40$ ratio, and (F) A $\beta 42 / T$-tau ratio.

proposed as markers demonstrating improvement in AD pathology $(54,55)$. Studies have shown that greater A $\beta$ load, indicated by lower CSF A $\beta 42$ concentration and increased $\mathrm{A} \beta \mathrm{PET}$, correlates with greater $\mathrm{WMH}$ burden (56-59); however, the findings regarding $p$-tau and total tau are less consistent, with some studies reporting correlations $(18,60)$ while other do not (59, $61,62)$. In the parent study, insulin treatment was associated with improved (increased) $A \beta 42 / A \beta 40$ and $A \beta 42 /$ tau ratios (13) for the secondary cohort treated with the device which was associated with cognitive benefit. The present study adds to these findings by demonstrating that WMHV changes inversely correlate with changes in $\mathrm{A} \beta 40, \mathrm{~A} \beta 42, \mathrm{~A} \beta 42 / \mathrm{A} \beta 40$ ratio, and $\mathrm{A} \beta 42$ / $\mathrm{t}$-tau ratio; however, these associations differ by region and CSF metric. The relationship between WMHV and tau and $A \beta$ may change throughout the course of the disease as $A \beta$ pathology is stabilized and tau pathology increases; however, this hypothesis is still in contention (59). Insulin affects the regulation of both $A \beta$ and hyperphosphorylated tau (63). Both $A \beta 40$ (64) and A $\beta 42$ (65) cause inflammation via production of reactive oxygen species. A $\beta 42$ aggregates and starts seeding points which grow to plaques leading to death in several cell types including oligodendrocytes $(66,67)$, while $A \beta 40$ is more prone to form deposits on vessels walls where it damages pericytes $(68,69)$. Hyperphosphorylated tau also leads to neurovascular dysfunction resulting in reduced energy supply due to aberrant vessel dilation (70). Insulin may act indirectly to stop WMH progression by preventing damage caused by hyperphosphorylated tau, $\mathrm{A} \beta 40$, and $\mathrm{A} \beta 42$ to both the blood brain barrier and myelin producing oligodendrocytes. Further research needs to be done to elucidate patterns behind regional $\mathrm{WMH}$ load and CSF AD biomarkers of $\mathrm{A} \beta$ and tau.

Numerous studies have sought to identify the temporal and spatial patterns of gray matter volume loss in typical AD progression (71). While there is not a consensus across all studies, several vulnerable regions have been highlighted $(11,72)$. Measures of gray matter volume and thickness were unchanged by 12 months of INI administration. It is possible that our region of interest, while widely accepted, failed to capture subtle differences between treatment groups. Other regions of the brain that were excluded from our analysis may be more sensitive to changes specific to this intervention. Previous interventions have shown decreased rates of atrophy in patients with MCI treated with other noninsulin interventions; however, these findings were over a 2 -year period (73). It is possible that our intervention was not long enough to detect subtle changes in gray matter volume. Cognitive improvement has been observed 
without halting gray matter atrophy. A phase II clinical trial testing daily administration of resveratrol showed greater reduction in brain volume in the treatment group compared to placebo after one year of treatment, but the resveratrol-treated group also showed less decline on the ADCS-ADL suggesting interventions can still be beneficial independent of apparent reduction of gray matter volume (74).

Our study had several limitations. Our small sample size may have contributed to our inability to detect preservation of gray matter volume and cortical thickness by INI treatment, and may also have reduced our ability to detect relationships between WMHVs and other measures. Our cohort was predominantly white $(93 \%)$ and male $(62 \%)$ and thus results may not generalize to a more diverse population. A number of participants did not complete the trial or had usable data at either baseline or follow up. These missing datapoints could result in a completer bias; however, this is unlikely as those with incomplete data did not differ demographically or in any baseline measures from completers. It is also possible that a longer intervention may be needed to observe a divergence between groups on our measures. These results need to be replicated in a larger, longer study in order to determine the effects of insulin on the brain WM health and the mechanistic pathways underlying these effects.

In conclusion, we found that treating $\mathrm{MCI}$ and $\mathrm{AD}$ patients with 12 months of INI significantly reduced WMH progression without affecting gray matter volume or cortical thickness, and that increases in WMHV correlated with both worsening in AD CSF biomarker profile and cognitive/functional measures. These findings support insulin's potential as a therapeutic option for AD; however, more research needs to be conducted to elucidate mechanism through which insulin may impact white matter integrity.

Funding and acknowledgments: This study was supported by the National Institute on Aging (NIA RF1AG041845). Eli Lilly provided diluent placebo for the blinded phase of the trial, and insulin for the open label portion at no cost; they had no input into the design, conduct or interpretation of trial results or in the preparation of this manuscript. The authors would like to thank the site personnel and the following Site Prinicipal Investigators for their perseverance throughout the trial: Deniz Erten-Lyons, David Knopman, Joseph Kass, Rachelle Doody, Hillel Grossman, Neelum Aggarwal, Esther Oh, Gregory Jicha, Anton Porsteinsson, Aimee Pierce, Gaby Thai, Ruth Mulnard, Allan Levey, Jeffrey Burns, Neill GraffRadford, Jared Brosch, Martin Farlow, Christopher van Dyck, Marek-Marsel Mesulam, Ian Grant, Raymond Scott Turner, Scott McGinnis, Thomas Obisesan Alan Lerner, Allison Perrin, Laura Baker, Elaine Peskind, Henry Querfurth, Brian Ott, Ralph Richter, Jacobo Mintzer, Marwan Sabbagh, Jiong Shi, Daniel Press, Shauna Yuan, David Carr, Nupar Ghoshal, Amanda Smith, James Galvin, and Kyle Womack. We are particularly grateful to the trial participants for their dedication.

Conflict of interest: The authors have no conflict of interest to report.

Ethical Standards: The study was approved by the Institutional Review Boards of all participating institutions. All participants provided written informed consent to participate in the study.

Open Access: This article is distributed under the terms of the Creative Commons Attribution 4.0 International License (http:/ / creativecommons.org/ licenses/by/4.0/), which permits use, duplication, adaptation, distribution and reproduction in any medium or format, as long as you give appropriate credit to the original author(s) and the source, provide a link to the Creative Commons license and indicate if changes were made.

\section{References}

1. Association, A.s., 2019 Alzheimer's disease facts and figures. Alzheimer's \& Dementia, 2019. 15(3): p. 321-387.

2. Sevigny, J., P. Chiao, T. Bussiere, et al., The antibody aducanumab reduces Abeta plaques in Alzheimer's disease. Nature, 2016. 537(7618): p. 50-6.

3. Klein, G., P. Delmar, G.A. Kerchner, et al., Thirty-Six-Month Amyloid Positron Emission Tomography Results Show Continued Reduction in Amyloid Burden with Subcutaneous Gantenerumab. J Prev Alzheimers Dis, 2021. 8(1): p. 3-6.

4. La Joie, R., A. Bejanin, A.M. Fagan, et al., Associations between [(18)F]AV1451 tau PET and CSF measures of tau pathology in a clinical sample. Neurology, 2018. 90(4): p. e282-e290.

5. Schipke, C.G., N. Koglin, S. Bullich, et al., Correlation of florbetaben PET imaging and the amyloid peptide Ass42 in cerebrospinal fluid. Psychiatry Res Neuroimaging, 2017. 265: p. 98-101.

6. Olsson, B., R. Lautner, U. Andreasson, et al., CSF and blood biomarkers for the diagnosis of Alzheimer's disease: a systematic review and meta-analysis. Lancet Neurol, 2016. 15(7): p. 673-684

7. Hansson, O., S. Lehmann, M. Otto, H. Zetterberg, and P. Lewczuk, Advantages and disadvantages of the use of the CSF Amyloid beta (Abeta) 42/40 ratio in the diagnosis of Alzheimer's Disease. Alzheimers Res Ther, 2019. 11(1): p. 34.

8. Blennow, K. and H. Hampel, CSF markers for incipient Alzheimer's disease. Lancet Neurol, 2003. 2(10): p. 605-13.

9. Driscoll, I., C. Davatzikos, Y. An, et al., Longitudinal pattern of regional brain volume change differentiates normal aging from MCI. Neurology, 2009. 72(22): p. 1906-13.

10. Jack, C.R., Jr., F. Barkhof, M.A. Bernstein, et al., Steps to standardization and validation of hippocampal volumetry as a biomarker in clinical trials and diagnostic criterion for Alzheimer's disease. Alzheimers Dement, 2011. 7(4): p 474-485 e4.

11. Schwarz, C.G., J.L. Gunter, H.J. Wiste, et al., A large-scale comparison of cortical thickness and volume methods for measuring Alzheimer's disease severity. Neuroimage Clin, 2016. 11: p. 802-812.

12. Sur, C., J. Kost, D. Scott, et al., BACE inhibition causes rapid, regional, and non-progressive volume reduction in Alzheimer's disease brain. Brain, 2020.

13. Craft, S., R. Raman, T.W. Chow, et al., Safety, Efficacy, and Feasibility of Intranasal Insulin for the Treatment of Mild Cognitive Impairment and Alzheimer Disease Dementia: A Randomized Clinical Trial. JAMA Neurol, 2020. 77(9): p. 1099-1109.

14. Raman, M.R., K. Kantarci, M.E. Murray, C.R. Jack, Jr., and P. Vemuri, Imaging markers of cerebrovascular pathologies: Pathophysiology, clinical presentation, and risk factors. Alzheimers Dement (Amst), 2016. 5: p. 5-14.

15. Abraham, H.M., L. Wolfson, N. Moscufo, C.R. Guttmann, R.F. Kaplan, and W.B. White, Cardiovascular risk factors and small vessel disease of the brain: Blood pressure, white matter lesions, and functional decline in older persons. J Cereb Blood Flow Metab, 2016. 36(1): p. 132-42.

16. Roseborough, A., J. Ramirez, S.E. Black, and J.D. Edwards, Associations between amyloid beta and white matter hyperintensities: A systematic review. Alzheimers Dement, 2017. 13(10): p. 1154-1167.

17. Villeneuve, S. and W.J. Jagust, Imaging Vascular Disease and Amyloid in the Aging Brain: Implications for Treatment. J Prev Alzheimers Dis, 2015. 2(1): p. 64-70.

18. McAleese, K.E., M. Firbank, M. Dey, et al., Cortical tau load is associated with white matter hyperintensities. Acta Neuropathol Commun, 2015. 3: p. 60.

19. Thal, D.R., The pre-capillary segment of the blood-brain barrier and its relation to perivascular drainage in Alzheimer's disease and small vessel disease. ScientificWorldJournal, 2009. 9: p. 557-63.

20. Iturria-Medina, Y, R.C. Sotero, P.J. Toussaint, J.M. Mateos-Perez, A.C. Evans, and I. Alzheimer's Disease Neuroimaging, Early role of vascular dysregulation on late-onset Alzheimer's disease based on multifactorial data-driven analysis. Nat Commun, 2016. 7: p. 11934.

21. Honjo, K., S.E. Black, and N.P. Verhoeff, Alzheimer's disease, cerebrovascular disease, and the beta-amyloid cascade. Can J Neurol Sci, 2012. 39(6): p. 712-28.

22. Snyder, H.M., R.A. Corriveau, S. Craft, et al., Vascular contributions to cognitive impairment and dementia including Alzheimer's disease. Alzheimers Dement, 2015. 11(6): p. 710-7.

23. Yamada, M., Predicting cerebral amyloid angiopathy-related intracerebral hemorrhages and other cerebrovascular disorders in Alzheimer's disease. Front Neurol, 2012. 3: p. 64

24. Wardlaw, J.M., E.E. Smith, G.J. Biessels, et al., Neuroimaging standards for research into small vessel disease and its contribution to ageing and neurodegeneration. The Lancet Neurology, 2013. 12(8): p. 822-838.

25. van den Heuvel, D.M., V.H. ten Dam, A.J. de Craen, et al., Measuring longitudinal white matter changes: comparison of a visual rating scale with a volumetric measurement. AJNR Am J Neuroradiol, 2006. 27(4): p. 875-8.

26. Kapeller, P., R. Barber, R.J. Vermeulen, et al., Visual rating of age-related white matter changes on magnetic resonance imaging: scale comparison, interrater agreement, and correlations with quantitative measurements. Stroke, 2003. 34(2): p. 441-5. 
27. Ramirez, J., A.A. McNeely, C.J.M. Scott, M. Masellis, S.E. Black, and I. Alzheimer's Disease Neuroimaging, White matter hyperintensity burden in elderly cohort studies: The Sunnybrook Dementia Study, Alzheimer's Disease Neuroimaging Initiative, and Three-City Study. Alzheimers Dement, 2016. 12(2): p. 203-210.

28. Rizvi, B., A. Narkhede, B.S. Last, et al., The effect of white matter hyperintensities on cognition is mediated by cortical atrophy. Neurobiol Aging, 2018. 64: p. 25-32.

29. DeCarli, C., E. Fletcher, V. Ramey, D. Harvey, and W.J. Jagust, Anatomica mapping of white matter hyperintensities $(\mathrm{WMH})$ : exploring the relationships between periventricular WMH, deep WMH, and total WMH burden. Stroke, 2005. 36(1): p. 50-5.

30. Lindemer, E.R., D.N. Greve, B. Fischl, J.C. Augustinack, D.H. Salat, and I Alzheimer's Disease Neuroimaging, Differential Regional Distribution of Juxtacortical White Matter Signal Abnormalities in Aging and Alzheimer's Disease. J Alzheimers Dis, 2017. 57(1): p. 293-303.

31. Guzman, V.A., O.T. Carmichael, C. Schwarz, et al., White matter hyperintensities and amyloid are independently associated with entorhinal cortex volume among individuals with mild cognitive impairment. Alzheimers Dement, 2013. 9(5 Suppl): p. S124-31.

32. Lee, S., F. Viqar, M.E. Zimmerman, et al., White matter hyperintensities are a core feature of Alzheimer's disease: Evidence from the dominantly inherited Alzheimer network. Ann Neurol, 2016. 79(6): p. 929-39.

33. Garnier-Crussard, A., S. Bougacha, M. Wirth, et al., White matter hyperintensities across the adult lifespan: relation to age, Abeta load, and cognition. Alzheimers Res Ther, 2020. 12(1): p. 127.

34. Birdsill, A.C., R.L. Koscik, E.M. Jonaitis, et al., Regional white matter hyperintensities: aging, Alzheimer's disease risk, and cognitive function. Neurobiol Aging, 2014. 35(4): p. 769-76.

35. Taylor, W.D., D.C. Steffens, J.R. MacFall, et al., White matter hyperintensity progression and late-life depression outcomes. Arch Gen Psychiatry, 2003 60(11): p. 1090-6.

36. McNay, E.C. and J. Pearson-Leary, GluT4: A central player in hippocampal memory and brain insulin resistance. Exp Neurol, 2020. 323: p. 113076

37. Muhic, M., N. Vardjan, H.H. Chowdhury, R. Zorec, and M. Kreft, Insulin and Insulin-like Growth Factor 1 (IGF-1) Modulate Cytoplasmic Glucose and Glycogen Levels but Not Glucose Transport across the Membrane in Astrocytes. J Biol Chem, 2015. 290(17): p. 11167-76.

38. de la Monte, S.M., Insulin resistance and Alzheimer's disease. BMB Rep, 2009 42(8): p. 475-81.

39. Arnold, S.E., Z. Arvanitakis, S.L. Macauley-Rambach, et al., Brain insulin resistance in type 2 diabetes and Alzheimer disease: concepts and conundrums. Nat Rev Neurol, 2018. 14(3): p. 168-181.

40. Suzanne, M., Insulin resistance and neurodegeneration: progress towards the development of new therapeutics for Alzheimer's disease. Drugs, 2017. 77(1): p. 47-65.

41. Lochhead, J.J., D.J. Wolak, M.E. Pizzo, and R.G. Thorne, Rapid transport within cerebral perivascular spaces underlies widespread tracer distribution in the brain after intranasal administration. J Cereb Blood Flow Metab, 2015 35(3): p. 371-81.

42. Born, J., T. Lange, W. Kern, G.P. McGregor, U. Bickel, and H.L. Fehm, Sniffing neuropeptides: a transnasal approach to the human brain. Nat Neurosci, 2002 5(6): p. 514-6

43. Craft, S., L.D. Baker, T.J. Montine, et al., Intranasal insulin therapy for Alzheimer disease and amnestic mild cognitive impairment: a pilot clinical trial. Arch Neurol, 2012. 69(1): p. 29-38.

44. de la Monte, S.M. and P. Grammas, Insulin Resistance and Oligodendrocyte/ Microvascular Endothelial Cell Dysfunction as Mediators of White Matter Degeneration in Alzheimer's Disease, in Alzheimer's Disease, T. Wisniewski, Editor. 2019: Brisbane (AU).

45. Ashrafian, H., E.H. Zadeh, and R.H. Khan, Review on Alzheimer's disease: Inhibition of amyloid beta and tau tangle formation. Int J Biol Macromol, 2020 167: p. 382-394.

46. Schmidt, P., C. Gaser, M. Arsic, et al., An automated tool for detection of FLAIR-hyperintense white-matter lesions in Multiple Sclerosis. Neuroimage, 2012. 59(4): p. 3774-83.

47. Schwarz, C., J. Gunter, C. Ward, et al. The mayo clinic adult lifespan template (MCALT): better quantification across the lifespan. in Alzheimer's Association International Conference.[Google Scholar]. 2017.

48. Jack, C.R., Jr., H.J. Wiste, T.M. Therneau, et al., Associations of Amyloid, Tau, and Neurodegeneration Biomarker Profiles With Rates of Memory Decline Among Individuals Without Dementia. JAMA, 2019. 321(23): p. 2316-2325.

49. Brickman, A.M., F.A. Provenzano, J. Muraskin, et al., Regional white matter hyperintensity volume, not hippocampal atrophy, predicts incident Alzheimer disease in the community. Arch Neurol, 2012. 69(12): p. 1621-7.

50. Tubi, M.A., F.W. Feingold, D. Kothapalli, et al., White matter hyperintensities and their relationship to cognition: Effects of segmentation algorithm. Neuroimage, 2020. 206: p. 116327.
51. Gouw, A.A., A. Seewann, W.M. van der Flier, et al., Heterogeneity of small vessel disease: a systematic review of MRI and histopathology correlations. J Neurol Neurosurg Psychiatry, 2011. 82(2): p. 126-35.

52. Carmichael, O., C. Schwarz, D. Drucker, et al., Longitudinal changes in white matter disease and cognition in the first year of the Alzheimer disease neuroimaging initiative. Arch Neurol, 2010. 67(11): p. 1370-8.

53. Heo, J.H., S.T. Lee, C. Kon, H.J. Park, J.Y. Shim, and M. Kim, White matter hyperintensities and cognitive dysfunction in Alzheimer disease. J Geriatr Psychiatry Neurol, 2009. 22(3): p. 207-12.

54. Mitchell, A.J., CSF phosphorylated tau in the diagnosis and prognosis of mild cognitive impairment and Alzheimer's disease: a meta-analysis of 51 studies. J Neurol Neurosurg Psychiatry, 2009. 80(9): p. 966-75.

55. Hampel, H., A. Goernitz, and K. Buerger, Advances in the development of biomarkers for Alzheimer's disease: from CSF total tau and Abeta(1-42) proteins to phosphorylated tau protein. Brain Res Bull, 2003. 61(3): p. 243-53.

56. Kandel, B.M., B.B. Avants, J.C. Gee, et al., White matter hyperintensities are more highly associated with preclinical Alzheimer's disease than imaging and cognitive markers of neurodegeneration. Alzheimers Dement (Amst), 2016. 4: p. 18-27.

57. Marnane, M. and G.Y. Hsiung, Could Better Phenotyping Small Vessel Disease Provide New Insights into Alzheimer Disease and Improve Clinical Trial Outcomes? Curr Alzheimer Res, 2016. 13(7): p. 750-63.

58. Pietroboni, A.M., T. Carandini, A. Colombi, et al., Amyloid PET as a marker of normal-appearing white matter early damage in multiple sclerosis: correlation with CSF beta-amyloid levels and brain volumes. Eur J Nucl Med Mol Imaging, 2019. 46(2): p. 280-287.

59. Walsh, P., C.H. Sudre, C.M. Fiford, et al., CSF amyloid is a consistent predictor of white matter hyperintensities across the disease course from aging to Alzheimer's disease. Neurobiol Aging, 2020. 91: p. 5-14

60. Tosto, G., M.E. Zimmerman, J.L. Hamilton, O.T. Carmichael, A.M. Brickman and I. Alzheimer's Disease Neuroimaging, The effect of white matter hyperintensities on neurodegeneration in mild cognitive impairment. Alzheimers Dement, 2015. 11(12): p. 1510-1519.

61. Kester, M.I., J.D. Goos, C.E. Teunissen, et al., Associations between cerebral small-vessel disease and Alzheimer disease pathology as measured by cerebrospinal fluid biomarkers. JAMA Neurol, 2014. 71(7): p. 855-62.

62. Osborn, K.E., D. Liu, L.R. Samuels, et al., Cerebrospinal fluid beta-amyloid42 and neurofilament light relate to white matter hyperintensities. Neurobiol Aging, 2018. 68: p. 18-25.

63. Kellar, D. and S. Craft, Brain insulin resistance in Alzheimer's disease and related disorders: mechanisms and therapeutic approaches. Lancet Neurol, 2020. 19(9): p. 758-766.

64. Parks, J.K., T.S. Smith, P.A. Trimmer, J.P. Bennett, Jr., and W.D. Parker, Jr., Neurotoxic Abeta peptides increase oxidative stress in vivo through NMDA-receptor and nitric-oxide-synthase mechanisms, and inhibit complex IV activity and induce a mitochondrial permeability transition in vitro. J Neurochem, 2001. 76(4): p. 1050-6.

65. Kim, H.C., K. Yamada, A. Nitta, et al., Immunocytochemical evidence that amyloid beta (1-42) impairs endogenous antioxidant systems in vivo. Neuroscience, 2003. 119(2): p. 399-419.

66. Xu, J., S. Chen, S.H. Ahmed, et al., Amyloid-beta peptides are cytotoxic to oligodendrocytes. J Neurosci, 2001. 21(1): p. RC118.

67. Pak, K., S.L. Chan, and M.P. Mattson, Presenilin-1 mutation sensitizes oligodendrocytes to glutamate and amyloid toxicities, and exacerbates white matter damage and memory impairment in mice. Neuromolecular Med, 2003. 3(1): p. 53-64.

68. Verbeek, M.M., D.J. Ruiter, and R.M. de Waal, The role of amyloid in the pathogenesis of Alzheimer's disease. Biol Chem, 1997. 378(9): p. 937-50.

69. Schultz, N., K. Brannstrom, E. Byman, et al., Amyloid-beta 1-40 is associated with alterations in NG2+ pericyte population ex vivo and in vitro. Aging Cell, 2018. 17(3): p. e12728.

70. Park, L., K. Hochrainer, Y. Hattori, et al., Tau induces PSD95-neuronal NOS uncoupling and neurovascular dysfunction independent of neurodegeneration. Nat Neurosci, 2020. 23(9): p. 1079-1089.

71. Frisoni, G.B., N.C. Fox, C.R. Jack, Jr., P. Scheltens, and P.M. Thompson, The clinical use of structural MRI in Alzheimer disease. Nat Rev Neurol, 2010. 6(2): p. 67-77.

72. Wirth, M., A. Bejanin, R. La Joie, et al., Regional patterns of gray matter volume, hypometabolism, and beta-amyloid in groups at risk of Alzheimer's disease. Neurobiol Aging, 2018. 63: p. 140-151.

73. Smith, A.D., S.M. Smith, C.A. de Jager, et al., Homocysteine-lowering by $\mathrm{B}$ vitamins slows the rate of accelerated brain atrophy in mild cognitive impairment: a randomized controlled trial. PLoS One, 2010. 5(9): p. e12244

74. Turner, R.S., R.G. Thomas, S. Craft, et al., A randomized, double-blind, placebo-controlled trial of resveratrol for Alzheimer disease. Neurology, 2015. 85(16): p. 1383-91. 